\title{
Metastasis-associated gene 1 expression in human medulloblastoma and its association with invasion and metastasis in medulloblastoma Daoy cell lines
}

\author{
Y.S. Chen ${ }^{1,2}$, S.P. Li ${ }^{2}$, H. Xiao ${ }^{1}$, Z.Y. Xie', M.X. Tan ${ }^{2}$, B. Liu ${ }^{2}$ and \\ W.M. Zhang ${ }^{1}$ \\ ${ }^{1}$ Department of Neurosurgery, Zhujiang Hospital of Southern Medical University, \\ Guangzhou, Guangdong Province, China \\ ${ }^{2}$ Department of Neurosurgery, Dongguan People's Hospital, Dongguan, \\ Guangdong Province, China \\ Corresponding author: W.M. Zhang \\ E-mail: zhangwangming_2015@126.com \\ Genet. Mol. Res.15 (2): gmr.15027894 \\ Received October 22, 2015 \\ Accepted March 18, 2016 \\ Published June 17, 2016 \\ DOI http://dx.doi.org/10.4238/gmr.15027894
}

\begin{abstract}
This study aims to investigate the expression of metastasis-associated gene 1 (MTA1) in human medulloblastoma, and its significance in the invasion and metastasis in a medulloblastoma cell line. Positive expression rate of MTA1 protein in medulloblastoma and adjacent normal tissues collected from 29 medulloblastoma patients was detected by immunohistochemistry assay in vivo. In in vitro experiments, Daoy cells were transfected with MTA1-targeted small interfering RNA (siRNA, MTA1-siRNA group), niRNA (MTA1-niRNA group), and plasmid vectors (control group). Transfection efficiency was evaluated by PT-PCR and western blot; cell adhesion, migration, and invasion capacity was assessed by adhesion assays, scratch assays, and transwell chamber invasion assays, respectively. Results indicated that the positive expression rate of MTA1 protein in the medulloblastoma
\end{abstract}


tissues was higher as compared with that of the adjacent normal tissues $(\mathrm{P}<0.05)$. In addition, mRNA and protein expression of MTA1 in the MTA1-siRNA group was lower than that in the control and MTA1niRNA groups $(\mathrm{P}<0.05)$. Adhesion, migration, and invasion capacity of Daoy cells in the MTA1-siRNA group was inhibited as compared with the control and MTA1-niRNA groups $(\mathrm{P}<0.05)$. In conclusion, MTA1 expression was increased in medulloblastoma cells, while MTA1 knockdown in medulloblastoma cells inhibited MTA1 expression. In addition, MTA1 knockdown inhibited the adhesion, migration, and invasive capabilities of medulloblastoma cells. It is possible that MTA1 can serve as a biomarker and a potential therapeutic target for medulloblastoma.

Key words: MTA1; Medulloblastoma; Invasion; Metastasis; Adhesion; Immunohistochemistry

\section{INTRODUCTION}

Medulloblastoma is the most common form of malignant brain tumor in children and young adults. It originates in the cerebellum, posterior fossa, or near the fourth ventricle, and is a major cause of morbidity and mortality in pediatric oncology (Kool et al., 2012; Taylor et al., 2012). Local recurrences due to tumor cell migration and invasion is common in medulloblastoma (Sikkema et al., 2012). Medulloblastoma is mainly caused by increased intracranial pressure due to blockage of the fourth ventricle, and it commonly spreads along cerebral spine fluid pathways. Approximately $5 \%$ of medulloblastoma metastasize to a systemic location, especially to the long bone (Chen et al., 2011; Provasi et al., 2014). Surgery is currently the main treatment of choice for medulloblastoma patients; depending on the tumor stage and aggressiveness of tumor cells, medulloblastoma is also responsive to a variety of chemotherapies, radiation therapies, and combination therapies (Chai et al., 2015; Michiels et al., 2015). Despite recent advances in radiation and chemotherapies, the 5-year survival rate of medulloblastoma patients remains at less than 5\% (Cheng et al., 2014). Therefore, it is necessary to explore alternative treatment strategies to effectively control the recurrence and progression of this disease; one of our approaches is to control the invasion and metastasis of medulloblastoma.

Metastasis-associated proteins (MTA) is a family of ubiquitously expressed coregulators comprised of six gene products, including metastasis-associated gene 1 (MTA1), MTA1s, MTA1-ZG29p, MTA2, MTA3, and MTA3L, which originate from 3 different genes (Sen et al., 2014). MTA1 has emerged as one of the highly dysregulated oncogenes in human cancer, and elevated expression of this gene is correlated with aggressive tumors and unfavorable clinical outcomes in cancer patients (Zhang et al., 2015). In the past decade, MTA1 has been confirmed to have vital roles in transcription regulation. It modulates protein steady states by regulating protein ubiquitination, which leads to the DNA damage response. Studies have shown that it is overexpressed in various human hematologic and epithelial malignancies ( $\mathrm{Li}$ et al., 2012a). In addition, the critical role of MTA1 is highlighted in nucleosome remodeling and histone deacetylase $(\mathrm{NuRD})$ complexes, epithelial-mesenchymal transitions (EMT), DNA damage responses, oncogenesis, as well as transcription-dependent and -independent inflammations (Li et al., 2012b). Sen et al. (2014) reported that MTA1 
protein may be associated with carcinogenesis and tumor progression in different malignant cancers, suggesting that MTA1 protein can be an important tool for clinical diagnosis and treatment of cancers.

In the present study, we investigated the expression of MTA1 in human medulloblastoma by immunohistochemistry. In addition, we determined the significance of MTA1 expression in the invasion and metastasis of medulloblastoma by several in vitro assays in order the evaluate the underlying mechanisms of this cancer.

\section{MATERIAL AND METHODS}

\section{In vivo experiments}

\section{Study subjects}

Between December 2010 and December 2014, 29 patients with pathologically confirmed medulloblastoma who underwent surgical treatment were selected from the Department of Neurosurgery in Zhujiang Hospital of Southern Medical University and Dongguan People's Hospital. There were 15 men (51.7\%) and 14 women (48.3\%), ranging between 5 and 36 years of age (median age $=14$ years). Paraffin-embedded specimen tissues from potential study subjects were obtained. The inclusion criteria for patient recruitment were as follows: 1) medical records of patients were complete; 2) pathologic sampling was conducted, and all samples were confirmed to be medulloblastoma by the Department of Pathology; 3) total or partial resection was achieved in surgery. The exclusion criteria were as follows: 1) patients had incomplete clinical data; 2) patients with medulloblastoma were also affected by other primary tumors or had a history of other primary tumors. Daoy medulloblastoma cell line was purchased from American Tumor Cell Collection (ATCC). This study was approved by the Ethics Committee of Zhujiang Hospital of Southern Medical University and Dongguan People's Hospital. Written informed consents were obtained from all patients prior to study enrolment.

\section{Immunohistochemistry}

Streptavidin-peroxidase (SP) immunohistochemistry was performed, according to the manufacturer protocol. Tissues from the tumor resection were fixed in $10 \%$ neutralbuffered formalin, paraffin-embedded, sliced, de-waxed, and hydrated. Sodium citrate buffer $(\mathrm{pH}=6.0)$ was applied for heat-induced antigen retrieval, and $0.3 \% \mathrm{H}_{2} \mathrm{O}_{2}$ was used to block endogenous peroxidase activity for $10 \mathrm{~min}$. Tissues were incubated at $4^{\circ} \mathrm{C}$ overnight with primary anti-human MTA1 mouse polyclonal antibody (Abcam) at 1:200 dilution. On the second day, the tissue specimens were allowed to recover at room temperature for $30 \mathrm{~min}$, and were washed with phosphate buffered saline (PBS). Samples were then incubated with rabbit anti-rat secondary antibody (Beijing Zhongshan Jinqiao Biotechnology Co., Beijing, China) at $37^{\circ} \mathrm{C}$ for $30 \mathrm{~min}$. Tissues were again washed with $\mathrm{PBS}$, stained with diaminobenzidine (DAB, Beijing Zhongshan Jinqiao Biotechnology Co., Beijing, China), counterstained, and were finally mounted on glass slides. PBS was utilized as a negative control.

Tumor cells with pale brown or sepia particles were denoted as positive cells, using a semi-quantitative couple scoring method (Brown and Wahl, 1993). A score of 0 was given for no stain, 1 was given for low staining intensity (light yellow), and 2 for moderate/ 
high intensity (pale brown/sepia). The percentage of positive cells was then scored as 0 for unspecific staining of positive cells, 1 for $<20 \%$ positive cells, 2 for $20-50 \%$ positive cells, and 3 for $>50 \%$ positive cells. The staining results were divided into 2 grades of immune responses, (+) or (-). This was calculated as the sum of staining intensity (1) and percentage of positive cells (2). If $(1)<(2)$, the immune response was denoted as $(-)$; when $(1) \geq(2)$, the immune response was denoted as $(+)$.

\section{In vitro experiments}

\section{SiRNA-mediated downregulation of MTA1 expression in Daoy cells}

MTA1-targeted small interfering RNA(siRNA, target sequence: 5'-GAACATCTACGA CATCTCC-3') and nonsense control sequence niRNA were chemically synthesized (Qian et al., 2005). Daoy cells were transfected with MTA1-targeted siRNA (MTA1-siRNA group), niRNA (MTA1-niRNA group), and the plasmid vector Lipofectamine 2000 (Invitrogen) (control group).

Daoy cells were cultured in RPM- 1640 culture medium in at $37^{\circ} \mathrm{C}$ and $5 \% \mathrm{CO}_{2}$ until logarithmic growth phase was reached. Daoy cells were transfected with MTA1-siRNA and MTA1-niRNA using Lipofectamine 2000 (Invitrogen; Cytofectin ${ }^{\mathrm{TM}}$, Gene Therapy Systems). Cells transfected with the empty plasmid vector were used as the negative control. All procedures were performed according to the manufacturer instructions. Transfection results were observed 48-96 h following transfection procedures.

\section{RT-PCR}

Total RNA was isolated using Trizol (Invitrogen, Carlsbad, CA, USA). The purity and concentration of the isolated RNA were detected with an ultraviolet spectrophotometer (Analytik Jena AG, Germany). Total RNA ( $2 \mu \mathrm{g}$ ) was reverse transcribed using the avian myeloblastosis virus (AMV), and PCR amplification was performed using complementary DNA (cDNA, $5 \mu \mathrm{L}$ ). PCR conditions were as follows: denaturation at $94^{\circ} \mathrm{C}$ for $30 \mathrm{~s}$, annealing at $55^{\circ} \mathrm{C}$ for $40 \mathrm{~s}$, and extension at $72^{\circ} \mathrm{C}$ for $30 \mathrm{~s}$. The primers used were synthesized by Shanghai Sangon Biotechnology Co. (Shanghai, China). The forward primer sequence of MTA1 was 5'-CCGGGCCTGCGAGAGCTGTTACAC-3' and the reverse primer sequence was 5'-CACGGCTTCCAGCGGCTTGCGTAC-3'. The size of the amplified MTA1 fragment was $442 \mathrm{bp}$. The forward primer sequence of $\beta$-actin (internal reference gene) was 5'-GGCTACAGCTTCACCACCAC-3', and the reverse primer sequence was 5'-AGGAAGGAAGGCTGGAAGAG-3'. The size of the amplified $\beta$-actin fragment was 210 bp. The amplified products were analyzed by gel electrophoresis. The expression level of MTA1 mRNA was calculated as the absorbance ratio of the amplified MTA1 band to the $\beta$-actin band.

\section{Western blot assay}

Daoy cells were collected from each group, and cell proteins were extracted. Protein samples were transferred onto the membrane using a $12 \%$ polyacrylamide gel. The samples were sealed at room temperature with goat anti-human MTA1 polyclonal (Santa Cruz Biotechnology, Inc., Santa Cruz, CA, USA) and mouse anti-human $\beta$-actin monoclonal (Santa 
Cruz Biotechnology, Inc.) primary antibodies at 1:200 and 1:1000 dilutions, respectively. Samples were then incubated with the relevant secondary antibodies. Films were exposed in the dark, developed, and finally analyzed.

\section{Adhesion assay}

At $48 \mathrm{~h}$ following transfection, Daoy cells in good condition were digested by trypsin and seeded onto 96-well plates coated with $100 \mu \mathrm{g} / \mathrm{mL}$ Matrigel (Becton, Dickinson and Company, USA). Cells were plated at a density of $1 \times 10^{5}$ cells/well in triplicates. At $30 \mathrm{~min}$, $60 \mathrm{~min}$, and 90 min post- incubation, cells were washed with PBS to remove floating cells. 3-(4,5-dimethylthiazol-2-yl)-2,5-diphenyltetrazolium bromide assay was used to determine the light absorbance at $570 \mathrm{~nm}$ (A). The percentage of cells adherence was calculated as follows: adhesion rate $(\%)=\mathrm{A}_{\text {cells adhering to Matrigel }} / \mathrm{A}_{\text {total cells }} \times 100 \%$.

\section{Scratch assay}

At $48 \mathrm{~h}$ following transfection, Daoy cells in good condition were seeded into 6-well plates. The Daoy cells at the bottom of the plates were softly scratched on a rectilinear path with a sterile $200-\mu \mathrm{L}$ pipette tip, washed twice with PBS, and further cultured. At 8,16 , and $24 \mathrm{~h}$ following the scratch test, healing of the scratch wound was observed under an inverted microscope. Cell mobility was determined as the percentage of the cells that repopulated the scratch wound. At $0 \mathrm{~h}$, the percentage of healed cells was $0 \%$.

\section{Transwell chamber invasion assay}

Transwell chambers (Corning, New York, USA) were put into 24-well plates; 500 $\mu \mathrm{L}$ Dulbecco's modified Eagle's medium (DMEM) with 10\% calf serum was added into each lower chamber, and $200 \mu \mathrm{L}$ cell suspension was added into each upper chamber. Five wells were used for each group. At $12 \mathrm{~h}$ after routine culture, the transwell chambers were retrieved and fixed with $95 \%$ ethanol. The matrices on the inner surface of the chamber were then removed by cotton swabs, and fixed with $4 \%$ of paraformaldehyde. Cells were stained with hematoxylin-eosin (H\&E) and observed under a microscope.

\section{Statistical analysis}

The SPSS 19.0 software was used for statistical analysis. All measurement data were presented as mean \pm standard difference (SD). The means between the two groups were compared using the Student $t$-test; comparisons among multiple groups were conducted using the one-way analysis of variance (ANOVA). Enumeration data were presented as percentages or rates, and were analyzed by the $\chi^{2}$ test. All data were verified by the two-sided test; $\mathrm{P}<0.05$ was considered to be statistically significant.

\section{RESULTS}

\section{In vivo results}

\section{Staining evaluation}

Immunohistochemistry assay on MTA1 was performed in 29 medulloblastoma tissue 
specimens as well as the adjacent normal tissues. The results showed that the MTA1 protein is mainly expressed in the cell membrane and cytoplasm, and positive MTA1 immunoreactivity was found in the cell membrane and cytoplasm, as indicated by the pale brown color. In the adjacent normal tissues, the expression level of MTA1 was low. Overall, medulloblastoma tissues showed a greater number of cells with positive expression of the MTA1 protein with significantly increased staining intensity. The positive expression rate of MTA1 protein in the medulloblastoma tissues was higher as compared to that in the adjacent normal tissues (69.0 vs $10.3 \%, \mathrm{P}<0.001$ ) (Figure 1 and Table 1).

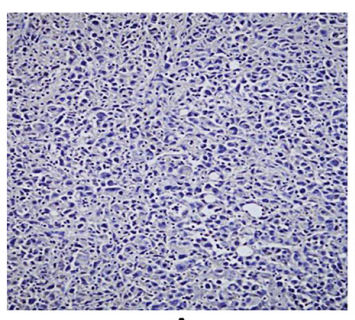

A

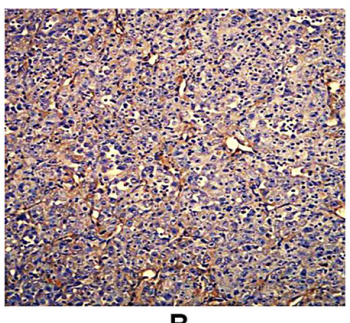

B

Figure 1. MTA1 protein expression in the medulloblastoma and normal tissues, as assessed by immunohistochemistry. A. Normal tissues. B. Medulloblastoma tissues; MTA1 = metastasis-associated protein-1.

Table 1. MTA1 protein expression in the medulloblastoma tissues and the adjacent normal tissues.

\begin{tabular}{l|c|c|c|c|c|c|c}
\hline Groups & $\mathrm{N}$ & - & + & ++ & +++ & Positive rate (\%) & $\mathrm{P}$ \\
\hline Medulloblastoma tissues & 29 & 9 & 4 & 11 & 5 & $69.0 \%$ & $<0.001$ \\
\hline Adjacent normal tissues & 29 & 26 & 2 & 1 & 0 & $10.3 \%$ & \\
\hline
\end{tabular}

MTA1 = metastasis-associated protein-1.

\section{MTA1 mRNA expression}

Expression level of MTA1 mRNA in the MTA1-siRNA group, the MTA1-niRNA group, and the control group were $0.398 \pm 0.057,0.976 \pm 0.089$, and $0.968 \pm 0.083$, respectively. Statistical analysis demonstrated a difference in MTA1 mRNA expression between the control group and the MTA1-niRNA group ( $>$ $>0.05)$. As expected, the expression level of MTA1 mRNA in the MTA1-siRNA group was lower than that in the control and MTA1-niRNA groups $(\mathrm{P}<0.05)$, demonstrating that the siRNA effectively inhibited the expression of MTA1 mRNA (Figure 2).

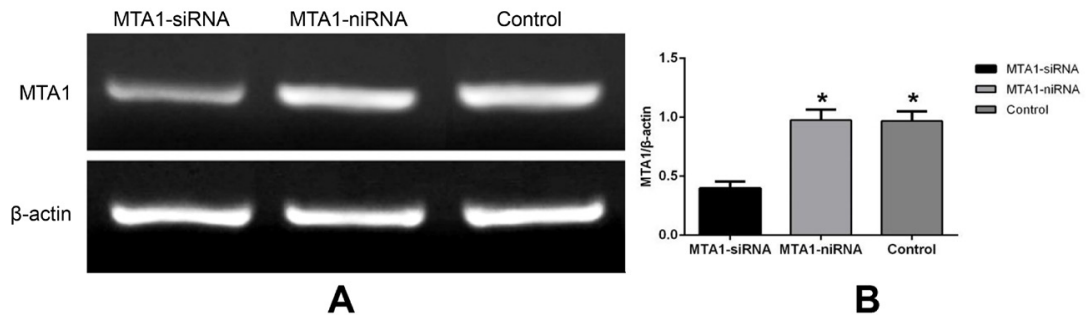

Figure 2. MTA1 mRNA expression in the MTA1-siRNA, MTA1-niRNA, and control groups, as assessed by RTPCR. MTA1 = metastasis-associated protein-1; siRNA = small-interfering RNA; RT-PCR = reverse transcriptionpolymerase chain reaction. A. MTA1 mRNA expression by RT-PCR. B. Expression levels of MTA1 mRNA. *P< 0.05 with respect to the MTA1-siRNA group. 


\section{MTA1 protein expression}

The protein expression of MTA1 in the control and MTA1-niRNA groups were $1.01 \pm$ 0.07 and $1.02 \pm 0.08$, respectively. In the MTA1-siRNA group, the expression level of MTA1 protein was clearly inhibited, with a relative expression level of $0.55 \pm 0.06$. As compared with the control and MTA1-niRNA groups, MTA1 protein expression in the MTA1-siRNA group was significantly reduced $(\mathrm{P}<0.05)$. No statistical difference in MTA1 protein expression was detected between the MTA1-niRNA group and the control group $(\mathrm{P}>0.05)$ (Figure 3).

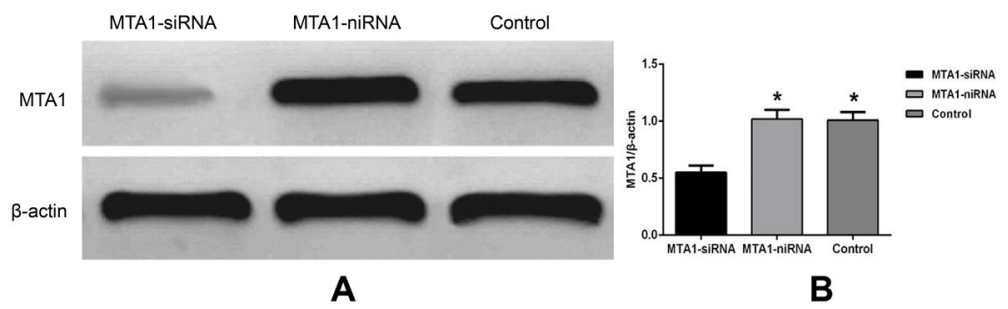

Figure 3. MTA1 protein expression in the MTA1-siRNA, MTA1-niRNA, and control groups, as assessed by western blot assay. MTA1 = metastasis-associated protein-1; siRNA $=$ small interfering RNA. A. MTA1 protein bands analyzed by western blot. B. Quantification of MTA1 protein expression; *P $<0.05$ with respect to the MTA1-siRNA group.

\section{In vitro results}

\section{Cell adhesion capacity}

The adhesion rate of Daoy cells in the MTA1-niRNA group, the control group, and the MTA1-siRNA group at 30,60 and 90 min post- incubation is presented in Figure 4. The results of in vitro cell adhesion analysis revealed that the adhesion rate of Daoy cells in the MTA1siRNA group at 30, 60, and 90 min after incubation was decreased as compared with those of the MTA1-niRNA and control groups $(\mathrm{P}<0.05)$. No statistical difference was observed in the adhesion rates of Daoy cells between the control group and the MTA1-niRNA group at 30, 60, and 90 min after incubation $(\mathrm{P}>0.05)$.

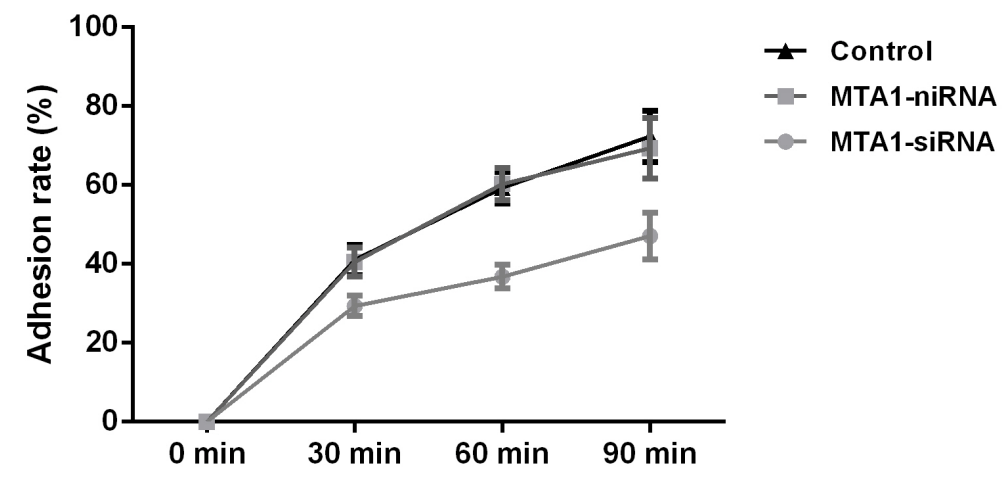

Figure 4. Comparison of cell adhesion capacities among the MTA1-siRNA, MTA1-niRNA, and control groups. MTA1 = metastasis-associated protein-1; siRNA = small interfering RNA. 


\section{Cell migration capacity}

Within $24 \mathrm{~h}$ after the scratch test, wound healing in the cell monolayer can be achieved through cell migration. Therefore, the scratch assay is an effective assessment of cell migration capacity. At $24 \mathrm{~h}$ after the scratch assay, we found that scratch wound healing gradually occurred in the MTA1-niRNA group and the control group. However, scratch wound healing in the MTA1-siRNA group was relative slow with incomplete healing. These findings suggested that the constructed siRNA effectively inhibited MTA1 expression, leading to reduced migration capacity and prolonged time requirement for scratch wound healing (Figure 5).

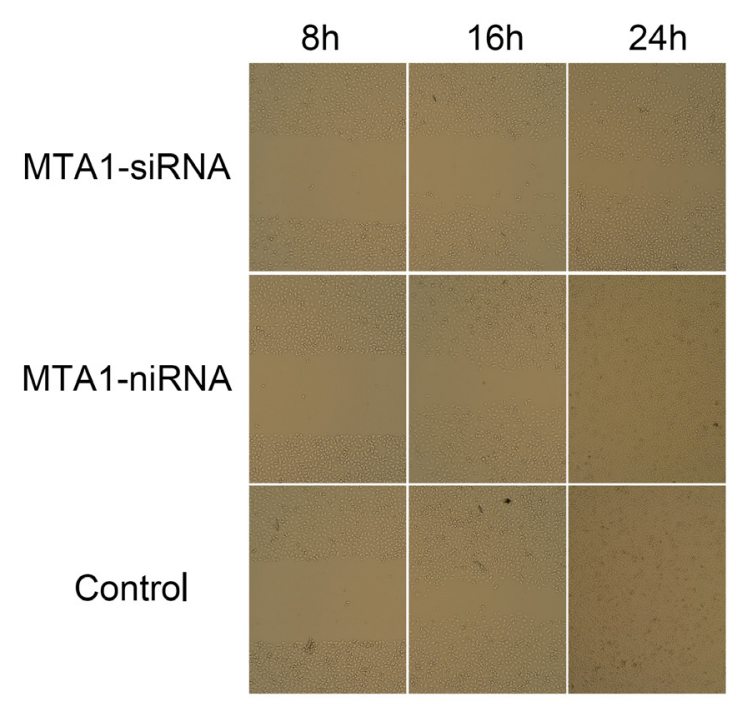

Figure 5. Comparison of cell migration capacities among the MTA1-siRNA, MTA1-niRNA, and control groups. MTA1 $=$ metastasis-associated protein $-1 ;$ siRNA $=$ small interfering RNA.

\section{Cell invasion capacity}

Matrigel could form structures similar to basement membranes on the surface of polycarbonate microfiltration membranes. Therefore, the invasion capacity of tumor cell could be determined by the cell's ability to invade through the Matrigel. The outcomes of the transwell chamber invasion assay revealed that Daoy cells in the control, MTA1niRNA, and MTA1-siRNA groups could invade through the microfiltration membrane coated with Matrigel. In the MTA1-siRNA group, the cell population that migrated through the microfiltration membrane was decreased as compared with the MTA1-niRNA and control groups $(202.5 \pm 15.6$ vs $479.8 \pm 22.3,461.9 \pm 23.6$, both $\mathrm{P}<0.05)$. No obvious difference in the cell population that migrated through the microfiltration membrane was detected between the control group and the MTA1-niRNA group $(\mathrm{P}>0.05)$. These outcomes indicated that MTA1 inhibition significantly reduced the invasive capacity of the Daoy medulloblastoma cell line (Figure 6). 


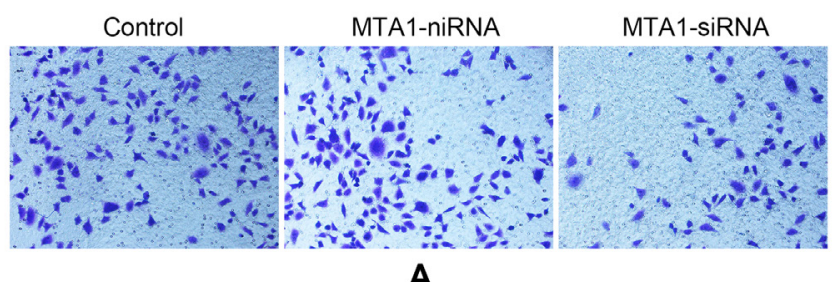

A

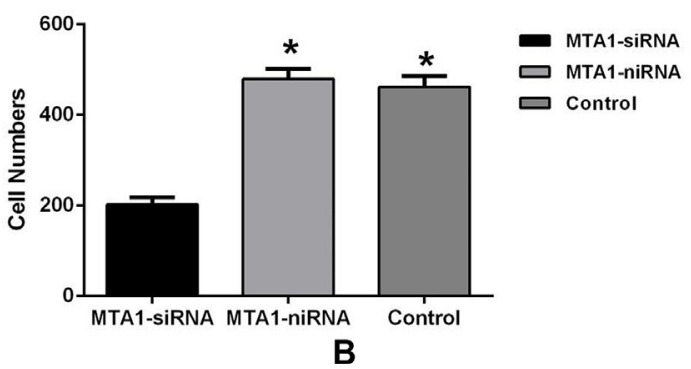

Figure 6. Comparison of cell invasion capacities among the MTA1-siRNA, MTA1-niRNA, and control groups. MTA1 = metastasis-associated protein-1; siRNA $=$ small interfering RNA. A. Representative images of cell invasion in the MTA1-siRNA, MTA1-niRNA, and control groups. B. Quantification of cells passing through the Matrigel gel.

\section{DISCUSSION}

Our present study evaluated the effect of in vitro MTA1 knockdown using MTA1siRNA in the Daoy medulloblastoma cell line. Prior to the establishment of the in vitro MTA1 knockdown model, we found higher MTA1 protein expression in the medulloblastoma tissues as compared with adjacent normal tissues via immunohistochemistry in vivo, suggesting that MTA1 protein expression may be associated with the onset of medulloblastoma. We therefore established an in vitro cell model of MTA1 knockdown, and found that MTA knockdown in tumor cells led to a reduction of MTA1 mRNA expression, demonstrating that the constructed MTA1-siRNA could effectively inhibit MTA1 mRNA expression. Moreover, using western blot assay, we also confirmed inhibition of MTA1 protein expression in the MTA knockdown tumor cells as compared with the cells in the MTA1-niRNA and control groups. The cancerrelated functions of MTA1 may result from one or more of the following potential mechanisms: first, posttranslational regulations of MTA1 in response to upstream signaling pathways; second, interactions of MTA1 with its binding proteins, including oncogenes; third, expression modulation of a large number of target genes due to its dual coregulatory activity (Sen et al., 2014). These mechanisms are further affected by dynamic changes in response to upstream signals, including growth factors, oncogenes, and genotoxic signals, as well as by the adaptive needs of cancer cells during oncogenesis and metastasis (Marzook et al., 2014). The findings in our present study are in line with several previous studies showing the importance of MTA family member overexpression in a number of human malignant cell types. Shan et al. (2015) have previously assessed the expression level of the MTA3 protein, and evaluated its relationship with the prognosis of glioma. He found decreased MTA3 expression in human glioma as well as its negative association to glioma prognosis. In a study by Cheng et al. (2014), it was found that MTA2 also plays a vital oncogenic role in the development and progression of gliomas. 
Furthermore, in vitro adhesion assays, scratch assays, and transwell chamber invasion assays showed that the adhesion, migration, and invasion capacity of medulloblastoma cells were significantly inhibited through MTA1 knockdown. Our results demonstrated that MTA1 plays a role in human medulloblastoma migration and invasion, and suggested that targeting MTA1 by MTA1-shRNA be a potential anticancer strategy in the treatment of medulloblastoma. Medulloblastoma cells are known to be not only highly proliferative, but can also readily invade surrounding brain structures (Kawauchi et al., 2012). A previous study showed that MTA2 inhibition reduced the invasion and metastasis of mammary tumor cells; the Mi2/ NuRD/MTA2 complex repressed the E-cadherin promoter and served as a twist-controlled cancer cell component that regulated EMT and metastasis (Fu et al., 2011). One previous study investigating the protein expression of MTA2 in non-small cell lung cancer (NSCLC) tissue samples reported that MTA2 is predominantly localized in the nucleus, and is associated with larger tumor sizes, advanced TNM stages, and lymph node metastasis (Liu et al., 2012). Aberrant expressions of MTA2 participate in the migration and invasion of gastric cancer cells when Sp1 binds to MTA2 promoters and enhances its transcriptional activity (Zhou et al., 2013). Based on the results of our present study, MTA1 knockdown reduced the migration and invasion of Daoy medulloblastoma cells, showing that the constructed MTA1-siRNA effectively decreased the expression of MTA1. Furthermore, it suggested that MTA1 may be associated with the migration and invasive capacity of medulloblastoma cells. Similarly, in a number of other cancers, MTA1 has also been shown to be associated with the migration and invasion of cells. For example, Li et al. (2013) have reported that MTA1 has antagonistic effects on NSCLC cell migration and invasion by targeting miR-125b. In addition, He et al. (2014) has documented that overexpression of MTA1 promotes invasiveness and metastasis of ovarian cancer cells, which may offer a novel strategy for ovarian cancer gene therapies.

In conclusion, evidence from our present study showed that protein expression of MTA1 was increased in medulloblastoma, while MTA1 knockdown in medulloblastoma cells inhibited MTA1 expression. We also demonstrated that MTA1 knockdown inhibited the adhesion, migration, and invasive abilities of medulloblastoma cells. Further investigations are required to elucidate the molecular mechanisms of MTA1 in regulating medulloblastoma tumorigenicity. Taken together, MTA1 expression may serve as a biomarker and a potential therapeutic target for medulloblastoma.

\section{Conflicts of interest}

The authors declare no conflicts of interest.

\section{ACKNOWLEDGMENTS}

We would like to acknowledge the reviewers for their helpful comments on this paper.

\section{REFERENCES}

Brown RS and Wahl RL (1993). Overexpression of Glut-1 glucose transporter in human breast cancer. An immunohistochemical study. Cancer 72: 2979-2985. http://dx.doi.org/10.1002/1097-0142(19931115)72:10<2979::AID CNCR2820721020>3.0.CO;2-X

Chai YH, Jung TY, Lee KH and Kim SK (2015). Progressive multiple cavernous angiomas after radiotherapy in a pediatric patient with medulloblastoma: a Case Report. Pediatr. Neurosurg. 50: 270-274. http://dx.doi.org/10.1159/000436977 
Chen B, Gao Y, Jiang T, Ding J, et al. (2011). Inhibition of tumor cell migration and invasion through knockdown of Rac1 expression in medulloblastoma cells. Cell. Mol. Neurobiol. 31: 251-257. http://dx.doi.org/10.1007/s10571010-9615-8.

Cheng CY, Chou YE, Ko CP, Yang SF, et al. (2014). Metastasis tumor-associated protein-2 knockdown suppresses the proliferation and invasion of human glioma cells in vitro and in vivo. J. Neurooncol. 120: 273-281. http://dx.doi. org/10.1007/s11060-014-1558-3.

Fu J, Qin L, He T, Qin J, et al. (2011). The TWIST/Mi2/NuRD protein complex and its essential role in cancer metastasis. Cell Res. 21: 275-289. http://dx.doi.org/10.1038/cr.2010.118.

He X, Zhou C, Zheng L and Xiong Z (2014). Overexpression of MTA1 promotes invasiveness and metastasis of ovarian cancer cells. Ir. J. Med. Sci. 183: 433-438. http://dx.doi.org/10.1007/s11845-013-1034-7.

Kawauchi D, Robinson G, Uziel T, Gibson P, et al. (2012). A mouse model of the most aggressive subgroup of human medulloblastoma. Cancer Cell 21: 168-180. http://dx.doi.org/10.1016/j.ccr.2011.12.023.

Kool M, Korshunov A, Remke M, Jones DT, et al. (2012). Molecular subgroups of medulloblastoma: an international meta-analysis of transcriptome, genetic aberrations, and clinical data of WNT, SHH, Group 3, and Group 4 medulloblastomas. Acta Neuropathol. 123: 473-484. http://dx.doi.org/10.1007/s00401-012-0958-8.

Li DQ, Pakala SB, Nair SS, Eswaran J, et al. (2012a). Metastasis-associated protein 1/nucleosome remodeling and histone deacetylase complex in cancer. Cancer Res. 72: 387-394. http://dx.doi.org/10.1158/0008-5472.CAN-11-2345.

Li SH, Tian H, Yue WM, Li L, et al. (2012b). Metastasis-associated protein 1 nuclear expression is closely associated with tumor progression and angiogenesis in patients with esophageal squamous cell cancer. World J. Surg. 36: 623-631. http://dx.doi.org/10.1007/s00268-011-1421-z.

Li Y, Chao Y, Fang Y, Wang J, et al. (2013). MTA1 promotes the invasion and migration of non-small cell lung cancer cells by downregulating miR-125b. J. Exp. Clin. Cancer Res. 32: 33. http://dx.doi.org/10.1186/1756-9966-32-33.

Liu SL, Han Y, Zhang Y, Xie CY, et al. (2012). Expression of metastasis-associated protein 2 (MTA2) might predict proliferation in non-small cell lung cancer. Target. Oncol. 7: 135-143. http://dx.doi.org/10.1007/s11523-012-0215-Z.

Marzook H, Deivendran S, Kumar R and Pillai MR (2014). Role of MTA1 in head and neck cancers. Cancer Metastasis Rev. 33: 953-964. http://dx.doi.org/10.1007/s10555-014-9521-5.

Michiels EM, Schouten-Van Meeteren AY, Doz F, Janssens GO, et al. (2015). Chemotherapy for children with medulloblastoma. Cochrane Database Syst. Rev. 1: CD006678.

Provasi J, Doyère V, Zélanti PS, Kieffer V, et al. (2014). Disrupted sensorimotor synchronization, but intact rhythm discrimination, in children treated for a cerebellar medulloblastoma. Res. Dev. Disabil. 35: 2053-2068. http://dx.doi. org/10.1016/j.ridd.2014.04.024.

Qian H, Lu N, Xue L, Liang X, et al. (2005). Reduced MTA1 expression by RNAi inhibits in vitro invasion and migration of esophageal squamous cell carcinoma cell line. Clin. Exp. Metastasis 22: 653-662. http://dx.doi.org/10.1007/ $\underline{\text { s10585-006-9005-2. }}$

Sen N, Gui B and Kumar R (2014). Role of MTA1 in cancer progression and metastasis. Cancer Metastasis Rev. 33: 879889. http://dx.doi.org/10.1007/s10555-014-9515-3.

Shan S, Hui G, Hou F, Shi H, et al. (2015). Expression of metastasis-associated protein 3 in human brain glioma related to tumor prognosis. Neurol. Sci. 36: 1799-1804. http://dx.doi.org/10.1007/s10072-015-2252-8.

Sikkema AH, den Dunnen WF, Hulleman E, van Vuurden DG, et al. (2012). EphB2 activity plays a pivotal role in pediatric medulloblastoma cell adhesion and invasion. Neuro-oncol. 14: 1125-1135. http://dx.doi.org/10.1093/neuonc/nos130

Taylor MD, Northcott PA, Korshunov A, Remke M, et al. (2012). Molecular subgroups of medulloblastoma: the current consensus. Acta Neuropathol. 123: 465-472. http://dx.doi.org/10.1007/s00401-011-0922-Z

Zhang H, Zhu X, Li N, Li D, et al. (2015). miR-125a-3p targets MTA1 to suppress NSCLC cell proliferation, migration, and invasion. Acta Biochim. Biophys. Sin. (Shanghai) 47: 496-503. http://dx.doi.org/10.1093/abbs/gmv039

Zhou C, Ji J, Cai Q, Shi M, et al. (2013). MTA2 promotes gastric cancer cells invasion and is transcriptionally regulated by Sp1. Mol. Cancer 12: 102. http://dx.doi.org/10.1186/1476-4598-12-102 